\title{
BMJ Open Use of tyrosine kinase inhibitors for paediatric Philadelphia chromosome- positive acute lymphoblastic leukaemia: a systematic review and meta-analysis
}

\author{
Min Chen, ${ }^{1,2}$ Yiping Zhu, ${ }^{2,3}$ Yunzhu Lin,, ${ }^{1,2}$ Tianzi Tengwang, ${ }^{4}$ Lingli Zhang (D) ${ }^{1,2}$
}

To cite: Chen M, Zhu Y, Lin Y, et al. Use of tyrosine kinase inhibitors for paediatric Philadelphia chromosomepositive acute lymphoblastic leukaemia: a systematic review and meta-analysis. BMJ Open 2021;11:e042814. doi:10.1136/ bmjopen-2020-042814

- Prepublication history and supplemental material for this paper is available online. To view these files, please visit the journal online (http://dx.doi. org/10.1136/bmjopen-2020042814).

Received 16 July 2020 Revised 02 0ctober 2020 Accepted 15 November 2020

Check for updates

(C) Author(s) (or their employer(s)) 2021. Re-use permitted under CC BY-NC. No commercial re-use. See rights and permissions. Published by BMJ.

For numbered affiliations see end of article.

Correspondence to

Dr Lingli Zhang;

zhlingli@sina.com

\section{ABSTRACT}

Objectives To investigate the effectiveness and safety of tyrosine kinase inhibitors (TKIs) in the management of paediatric Philadelphia chromosome-positive acute lymphoblastic leukaemia (Ph+ALL).

Design A systematic review and meta-analysis.

Data sources Electronic searches were conducted on CENTRAL, MEDLINE, EMBASE, SIOP, ASPHO, ASCO, ASH and four Chinese databases from inception to 8 March 2020. Language of publications was restricted in English and Chinese.

Eligibility criteria Prospective and retrospective comparative studies were included.

Data extraction and synthesis Two authors independently assessed and extracted data. Quality of studies was assessed by the Cochrane Collaboration's tool and Newcastle-Ottawa Scale. Subgroup analysis was performed by comparing different types of TKls. Quality of evidence was assessed using the Grading of Recommendations Assessment, Development and Evaluation approach.

Results Two randomised controlled trials (RCTs) and four cohort studies enrolling 536 patients were included. For RCTs, the pooled HR was $0.68(95 \% \mathrm{Cl} 0.26$ to 1.78$)$ in overall survival (OS), 0.63 (95\% Cl 0.28 to 1.42) in eventfree survival (EFS), respectively, comparing TKI arm with non-TKI arm for treatment of paediatric Ph+ALL. There was significant difference in OS and EFS between imatinib arm and dasatinib arm (HR 2.26, 95\% $\mathrm{Cl} 1.02$ to 5.01; HR 2.36; $95 \% \mathrm{Cl} 1.27$ to 4.39 , respectively). For cohort studies, the pooled HR was 0.25 (95\% Cl 0.14 to 0.47$)$ in $0 \mathrm{~S}, 0.25$ (95\% Cl 0.12 to 0.56 ) in EFS, respectively, comparing TKI arm with non-TKI arm. There was no significance difference in adverse drug reaction between TKI group and without TKI group (risk ratio (RR) $0.82,95 \% \mathrm{Cl} 0.63$ to 1.08 in RCT; RR $1.01,95 \%$ Cl 0.64 to 1.59 in cohort studies; respectively), and imatinib versus dasatinib (RR 0.97 , $95 \% \mathrm{Cl} 0.77$ to 1.23$)$. The quality of evidence was rated as low for OS, EFS and adverse drug reaction (ADR). Conclusions The combination of TKls with chemotherapy is likely to improve the OS and EFS rates in paediatric $\mathrm{Ph}+\mathrm{ALL}$, and dasatinib is superior than imatinib. Large sample size and prospective controlled studies are warranted.

PROSPERO registration number CRD42018104107.
Strengths and limitations of this study

- This study is the first systematic review and metaanalysis of the effectiveness and safety of TKIs in the management of paediatric $\mathrm{Ph}+\mathrm{ALL}$.

- The present review included both prospective and retrospective comparative studies.

- The Grading of Recommendations Assessment, Development and Evaluation approach was used to evaluate the quality of the evidence.

- This review was limited by the small number of studies and the heterogeneity of study design.

\section{INTRODUCTION}

Philadelphia chromosome-positive acute lymphoblastic leukaemia (Ph+ALL), which occurs in approximately $3 \%-5 \%$ of paediatric ALL patients, is recognised as a severe disease but leading to a dismal prognosis compared with those with Philadelphia chromosomenegative ALL. ${ }^{1}$

In the past decades, intensive chemotherapy has been used in the treatment of paediatric $\mathrm{Ph}+\mathrm{ALL}$ patients. ${ }^{23}$ Later, haematopoietic stem cell transplant (HSCT) in first complete remission (CR1) was considered the treatment of choice due to the improved rate of overall survival (OS) and event-free survival (EFS) than intensive chemotherapy alone. However, the mortality rate associated with transplantation was almost $50 \% .^{45}$ Recent studies suggested that tyrosine kinase inhibitors (TKIs) targeting BCR-ABL fusion protein combined with chemotherapy may be an alternative effective therapy. ${ }^{6}$ In adult patients with $\mathrm{Ph}+\mathrm{ALL}$, the use of TKIs in combination with chemotherapy was capable to increase the CR and HSCT rates and improve the early outcome. ${ }^{7}$ In paediatric patients, the Children's Oncology Group (COG) trial AALL0031 had shown 80\% 3-year EFS for Ph+ALL patients treated with intensive chemotherapy plus continuous $340 \mathrm{mg}$ / 
$\mathrm{m}^{2}$ imatinib. The addition of imatinib to chemotherapy has similar outcomes to HSCT, especially in patients who responded favourably. ${ }^{8}$ Long-term follow-up confirmed the favourable outcomes for these patients treated with imatinib plus intensive chemotherapy and HSCT seemed to be of no benefit. ${ }^{9}$ However, the trial was observational and confirmatory results are not yet available from the European intergroup study on post-induction treatment of Ph+ALL with imatinib trial. ${ }^{10}$

Newer TKIs including dasatinib and nilotinib were developed for those patients with resistance to imatinib. The resistance mechanisms are commonly associated with acquiring kinase domain mutations, reduced drug availability and activation of other signalling pathways such as the SRC family kinases. ${ }^{11}{ }^{12}$ Dasatinib is the more commonly used dual ABL/SRC kinase inhibitor. It can cross the blood-brain barrier to eradicate central nervous system leukaemia and play a role in most cases of imatinib resistance. ${ }^{13}{ }^{14}$ For patients with BCR-ABL T315I mutation $\mathrm{Ph}+\mathrm{ALL}$, the third-generation TKI ponatinib was reported as a valuable alternative treatment option. ${ }^{1516}$

Incorporation of TKIs into frontline regimens for patients with newly diagnosed Ph+ALLmay be the current general consensus. However, this view may not be universally accepted. It will be helpful to analyse systematically to determine if it is truly beneficial to add TKIs to conventional chemotherapy. Therefore, our objective is to investigate the current available evidence on the effectiveness and safety of TKIs in the management of paediatric $\mathrm{Ph}+\mathrm{ALL}$.

\section{METHODS}

This protocol was registered in the international PROSPERO register of systematic reviews.

\section{Patient and public involvement}

Patients and the public were not be involved in this review.

\section{Search strategy}

We searched the Cochrane Central Register of Controlled Trials (CENTRAL) published in the Cochrane Library, MEDLINE in PubMed, EMBASE (Ovid), the Chinese Biomedical Literature Database (CBM), Chinese National Knowledge Infrastructure (CNKI), VIP Database for Chinese Technical Periodicals (VIP) and WANFANG for English and Chinese references. The full search strategies used in this study were detailed in online supplemental file 1 . Retrieval time was from the inception of the database to 8 March 2020.

The following societies of conference proceedings of annual meetings (from the inception of TKIs treatment till 8 March 2020) were scanned :

1. International Society for Paediatric Oncology (SIOP).

2. American Society for Pediatric Hematology and Oncology (ASPHO).

3. American Society of Clinical Oncology (ASCO).

4. American Society of Hematology (ASH).
Inclusion and exclusion criteria

Study designs

There were limited randomised controlled trials (RCTs) in the treatment of paediatric patients with Ph+ALL; hence, we included retrospective and prospective cohort/case-control/randomised studies. Studies that did not include a comparative group of patients, duplicate studies, abstracts and historical control studies were excluded.

\section{Participants}

All patients aged $1-18$ years with $\mathrm{Ph}$ positive status diagnosed by cytogenetic or molecular were included. Participants with $\mathrm{Ph}$ negative at diagnosis but positive at relapse, more than two types of malignant neoplasm, relapse or refractory $\mathrm{Ph}+\mathrm{ALL}$ were excluded.

\section{Interventions}

TKIs alone, TKIs combined with other intervention.

\section{Comparators}

Treatment without TKIs.

\section{Outcomes}

1. OS, defined as time from start of treatment to death from any cause.

2. EFS, defined as time from start of treatment to an event, such as remission failure, relapse, death from any cause, abandonment of treatment, second malignancy or to the date of last follow-up contact.

3. Adverse drug reaction (ADR), collected and scored according to the National Cancer Institute Common Toxicity Criteria for Adverse Events (NCI-CTCAE) or other criterion by investigators and clinical research coordinators.

\section{Data extraction}

Literature search results were uploaded to EndNote V.X7. Two authors independently screened the title and abstract of all studies identified by the search strategy and obtained full articles for all potentially relevant trials. Disagreements were resolved by consensus between authors or achieved final resolution using a third-party arbitrator.

Two authors independently used a predesigned data collection form to extract data from each study. The items included: characteristics of the trial (author, country, publication year, design, etc), participants (age, sex, etc), interventions (dose, regimen, etc), outcomes and length of follow-up.

\section{Risk of bias in individual studies}

Two authors independently assessed the risk of bias using a standard form. For randomised trials, we used the domain-based evaluation recommended by the Cochrane Handbook for Systematic Reviews of Intervention to address the following domains: bias arising from the randomisation process, bias due to deviations from intended interventions, bias due to missing outcome data, 
bias in measurement of the outcome and bias in selection of the reported result. ${ }^{17}$ Plots of 'Risk of Bias' assessment were created using Review Manager V.5 (RevMan V.5). For non-randomised trials, we used the Newcastle-Ottawa Scale (NOS) items that were categorised into three broad perspectives: the selection of the study groups, the comparability of the groups and the ascertainment of either the exposure or outcome of interest for case-control or cohort studies, respectively. ${ }^{18}$ The maximum total score of NOS is 9 points.

\section{Data analysis and synthesis}

Strategy for data analysis

For dichotomous data, we used the risk ratio (RR) with 95\% CI as the effect measure. For time-to-event data, which take into account of the number and timing of events, we summarised and analysed data using HR with their corresponding 95\% CI. ${ }^{17}$ We used Parmar's method if HR was not explicitly presented in the research. ${ }^{19}$

\section{Assessment of heterogeneity}

We quantified heterogeneity using the $\mathrm{I}^{2}$ statistic, which illustrated the percentage of the variability in effect estimates resulting from heterogeneity. In the absence of significant heterogeneity $\left(\mathrm{I}^{2}\right.$ less than $50 \%$ ), we used a fixed-effect model for the estimation of treatment effect. ${ }^{17}$ Otherwise, we explored possible reasons for the occurrence of heterogeneity and used random-effects model.

\section{Data synthesis}

We entered data into the RevMan 5 software and made analyses according to the guidance provided in the Cochrane Handbook for Systematic Reviews of Interventions. ${ }^{20}$ We pooled results only if both treatment groups were comparable, including the outcome definition. Otherwise, we presented a narrative summary. If different analysis methods (such as intention-to-treat analysis, as-treated analysis) are used in the evaluation of benefits between groups, we adopt the results of the intention-totreat analysis to reduce bias.

\section{Assessment of reporting biases}

If we include 10 or more trials, we use funnel plots to assess biases including publication bias and other reporting biases. If there are biases, the funnel plots may be asymmetrical.

\section{Subgroup analysis}

Considering that different types of TKIs might affect the outcomes, we conducted a subgroup analysis.

\section{Grading the evidence}

We used the Grading of Recommendations Assessment, Development and Evaluation (GRADE) approach to rate the quality of evidence. ${ }^{21}$ In the GRADE approach, RCTs start as high-quality evidence. Five factors may lead to downgrading the quality of evidence. These factors include risk of bias, inconsistency, indirectness, imprecision and publication bias. Observational studies (including cohort and case-control studies) start as lowquality evidence. Three factors may lead to upgrading the quality of evidence, and these factors are large effect, dose response and all plausible residual confounding. The quality of the evidence was rated as high, moderate, low or very low. We used GRADEpro software (GRADEpro 2011) to create a 'Summary of findings' table for all three outcomes listed above.

\section{RESULTS}

\section{Study selection}

Our search strategy identified 2476 references from the electronic searches and 69 from other sources. Two hundred and fifty-two duplicate publications and 1928 articles were excluded after reviewing the title and abstract because they failed to meet the inclusion criteria such as type of article, study design, population or outcome of interest. Three hundred and sixty-five articles underwent full-text review and six literature were included in the final data analysis. The Preferred Reporting Items for Systematic Reviews and Meta-Analyses flow chart of bibliographic search was illustrated in figure 1 .

\section{Characteristics of included studies}

Six articles ${ }^{1022-26}$ (two prospective randomised open-label controlled trials and four retrospective cohort studies) were included in our analysis. Five studies ${ }^{1023-26}$ evaluated the efficacy and safety of TKIs in association with multidrug chemotherapy or chemotherapy alone in paediatric patients with Ph+ALL. Two of these studies 2526 came from the same research institution. Among them, one study ${ }^{25}$ retrospectively analysed 53 cases between 2008 and 2013. The other ${ }^{26}$ retrospectively analysed 92 cases between 2003 and 2012. One RCT ${ }^{22}$ compared the effect of dasatinib versus imatinib. Overall, 536 children aged 1-18 years with a confirmed diagnosis of $\mathrm{Ph}+\mathrm{ALL}$ contributed to the analysis. The chemotherapy regimen was modelled on Berlin-Frankfurt-Munster high-risk arm, the Chinese Childhood Leukemia Group ALL high-risk arm and the Chinese Children's Cancer Group ALL intermediate-risk arm. TKI drugs included firstgeneration imatinib and second-generation dasatinib. Patients were prescribed oral imatinib $\left(260-340 \mathrm{mg} / \mathrm{m}^{2} /\right.$ day) or dasatinib $\left(40-80 \mathrm{mg} / \mathrm{m}^{2} /\right.$ day) on day 8 , day 22 of remission induction or the end of induction. The exposure of TKIs ranged from 56 days to the end of therapy. Table 1 described the detailed characteristics of the studies included in this meta-analysis.

\section{Risk of bias assessment in included studies}

Figure 2 provided quality assessment of RCTs and table 2 described quality assessment of non-RCTs. In the two prospective trials, stratified randomisation was done centrally with web-based randomisation system and both were open label. Therefore, the random sequence generation was low risk while the blindness was high risk. All studies had low risk bias in incomplete outcome 


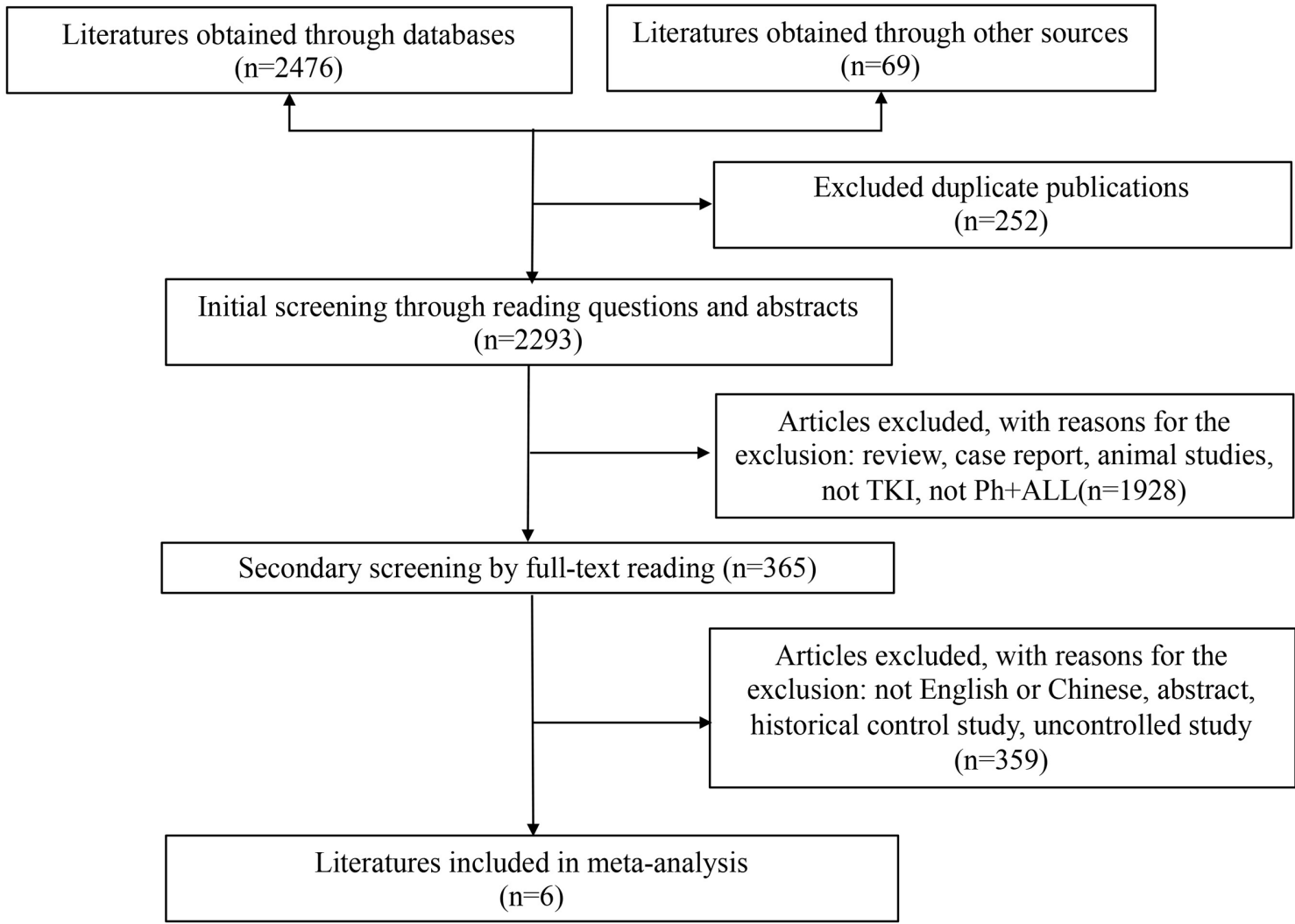

Figure 1 Preferred Reporting Items for Systematic Reviews and Meta-Analyses flow chart. This flow diagram illustrated the results of search and the process of screening and selecting studies for inclusion and the reasons for exclusions in this review. $\mathrm{Ph}+\mathrm{ALL}$,Philadelphia chromosome-positive acute lymphoblastic leukaemia; TKI, tyrosine kinase inhibitor.

data, selective reporting and other issues. In four cohort studies, the NOS methodological score was 7-8 points. In the selection section, both exposed cohort and unexposed cohort were drawn from the same source of participant. Data was collected and retrospectively reviewed from medical records. Outcome was not present at the beginning of all studies. In the comparability section, the three studies did not describe important confounding factors, except for TKI treatment between exposed and unexposed cohorts among paediatric Ph+ALL. In the outcome section, one study was followed up for 2 years, which might not be long enough for outcomes to occur. One study did not describe the patient's loss of follow-up. Overall, the retrospective research has highquality NOS scores. Therefore, the risk of bias of RCTs was rated as moderate and the cohort studies were rated as low.

\section{TKI versus non-TKI}

Overall survival

Four studies (one RCT, three cohort studies) ${ }^{10232426}$ with a total of 294 patients were included in the meta-analysis of OS for paediatric Ph+ALL patients. The pooled HR of OS in patients with TKI use was 0.68 (95\% CI 0.26 to 1.78, $\mathrm{p}=0.43$ ) in RCT and 0.25 (95\% CI 0.14 to $0.47, \mathrm{p}<0.05$ ) in cohort studies, respectively. Figure 3 showed the forest plot of the included studies.

\section{Event-free survival}

The primary outcome in one $\mathrm{RCT}^{10}$ was disease-free survival (DFS) because the group only included good-risk patients in CR1 and poor-risk patients were not included. Therefore, in this meta-analysis, DFS was considered equivalent to EFS. Four studies (one RCT, three cohort studies) ${ }^{10}{ }^{24-26}$ reported the EFS. Because Liu et $a l^{26}$ covered a longer period with larger number of patients, their results for EFS were included and those from Guo et $a l^{25}$ were excluded. Finally, a total of 200 patients were included. The pooled HR of EFS in Ph+ALL patients using TKI was 0.63 (95\% CI 0.28 to $1.42, \mathrm{p}=0.26$ ) in RCT and 0.25 (95\% CI 0.12 to $0.56, p<0.05)$ in cohort studies, respectively. Figure 4 showed the forest plot of the included studies.

\section{Adverse drug reaction}

Three studies (one RCT, two cohort studies) ${ }^{10} 2325$ reported serious ADR in patients with $\mathrm{Ph}+\mathrm{ALL}$. Heterogeneity in the cohort studies was significant $\left(\mathrm{I}^{2}=58 \%\right)$. We used a random-effect model for the estimation of treatment effect. In RCT, myelosuppression was the main cause of ADR. The proportions of patients with the most commonly reported serious ADR did not differ substantially between imatinib group and non-imatinib group $(\mathrm{p}=0.64)$. The most common serious ADR was infection, including fungal infection, localised infection and 


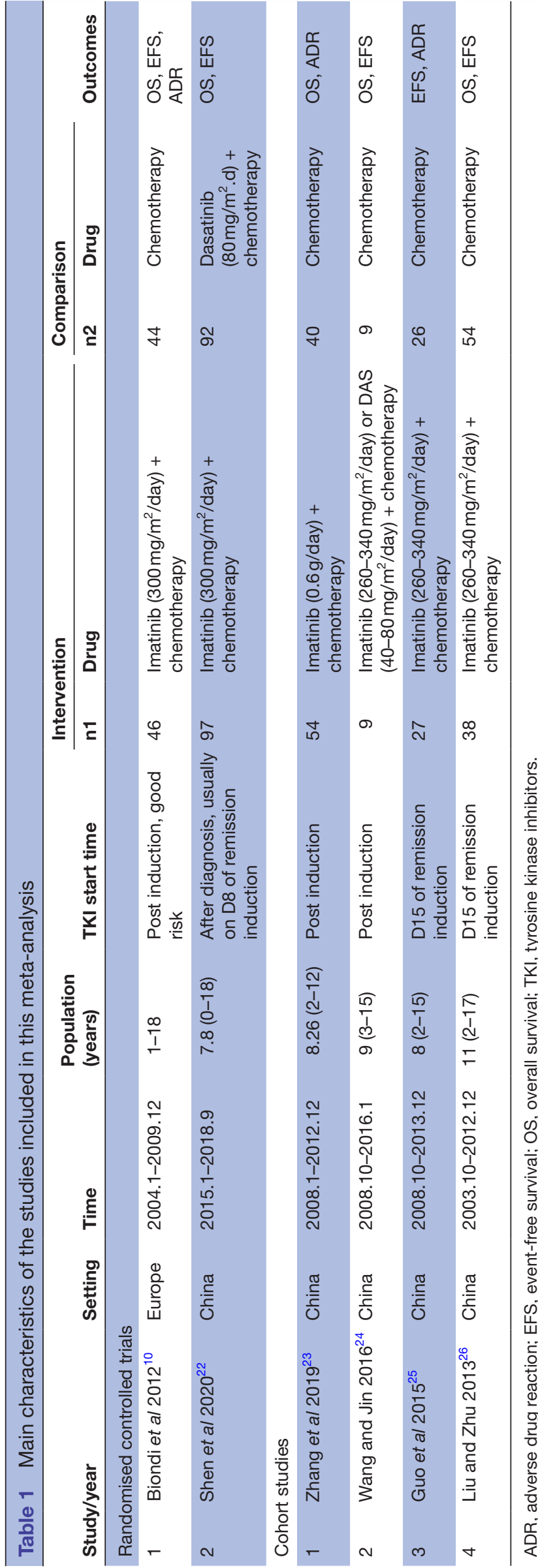

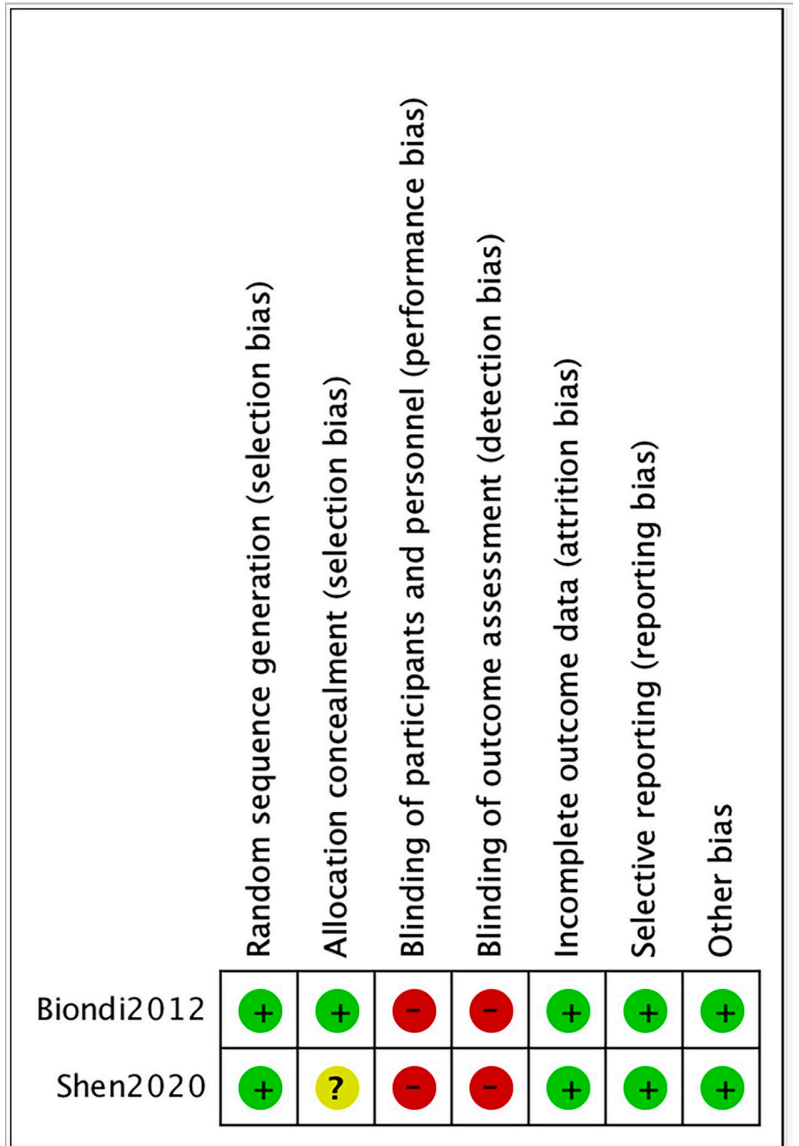

Figure 2 The quality assessment of randomised clinical trials. This plot is created by the software of RevMan V.5.3. It illustrated the quality of included randomised clinical trials with each of the judgement ('low risk', 'high risk' or 'unclear risk' of bias).

others. The pooled RR of serious ADR in patients with TKI use was 0.82 (95\% CI 0.63 to $1.08, \mathrm{p}=0.16$ ) in RCT. Result of the cohort studies was consistent with RCT. The pooled RR was 1.01 (95\% CI 0.64 to 1.59 , $\mathrm{p}=0.12$ ) (figure 5).

\section{Different TKls}

One RCT $^{22}$ with a total of $189 \mathrm{Ph}+\mathrm{ALL}$ children compared the OS, EFS and ADR of oral imatinib at a daily dosage of $300 \mathrm{mg} / \mathrm{m}^{2}$ versus dasatinib at a daily dosage of $80 \mathrm{mg} /$ $\mathrm{m}^{2}$, in combination with an intensive chemotherapy backbone. The HR of OS was 2.26 (95\% CI 1.02 to $5.01, \mathrm{p}=0.04)$. The HR of EFS was 2.36 (95\% CI 1.27 to $4.39 ; \mathrm{p}<0.05)$. There was no significant difference in the frequency of severe toxic effects between dasatinib and imatinib arms (RR $0.97 ; 95 \%$ CI 0.77 to $1.23, \mathrm{p}=0.81$ ). Infections constituted the most common serious adverse events. Approximately $5 \%$ of the patients in each arm died of fatal infections and $7 \%$ of patients in each treatment arm had disseminated fungal infections. The incidence of pleural effusion was $2 \%$ in the imatinib group and $4 \%$ in the dasatinib group. However, this difference was also not significant $(\mathrm{p}=0.44)$. 
Table 2 The quality assessment in cohort studies

\begin{tabular}{|c|c|c|c|c|c|}
\hline Study items & & Zhang et $a l^{23}$ & Wang and $\mathrm{Jin}^{24}$ & Guo et $a l^{25}$ & Liu and $\mathrm{Zhu}^{26}$ \\
\hline \multirow[t]{3}{*}{ Selection } & Representativeness of the exposed cohort & 1 & 1 & 1 & 1 \\
\hline & Selection of the non-exposed cohort & 1 & 1 & 1 & 1 \\
\hline & $\begin{array}{l}\text { Demonstration that outcome of interest was } \\
\text { not present at start of study }\end{array}$ & 1 & 1 & 1 & 1 \\
\hline \multirow[t]{3}{*}{ Outcome } & Assessment of outcome & 1 & 1 & 1 & 1 \\
\hline & $\begin{array}{l}\text { Was follow-up long enough for outcomes } \\
\text { to occur }\end{array}$ & 1 & 1 & 1 & 0 \\
\hline & Adequacy of follow-up of cohorts & 0 & 1 & 1 & 1 \\
\hline
\end{tabular}

NOS, Newcastle-Ottawa Scale.

\section{GRADE assessment}

The evidence provided by the RCTs was low quality for both comparison of TKI-based chemotherapy regimens versus chemotherapy alone, and the comparison of imatinib and dasatinib. The quality was downgraded by the risk of bias and imprecision due to high risk in blindness and wide $95 \%$ CI for effect estimates. Meanwhile, the evidence that the cohort studies provided to this review was of moderate quality. It was upgraded by the large magnitude of treatment effect. A summary of the GRADE assessment was shown in online supplemental file 2.

\section{DISCUSSION}

TKIs have revolutionised the management of $\mathrm{Ph}+\mathrm{ALL}$ patients. The National Comprehensive Cancer Network has recommended the use of TKI-based chemotherapy regimens for the adolescents and adults with Ph+ALL. ${ }^{7}$ Systematic review by Warraich et al comprising 18 prospective and retrospective studies with 462 participates showed that utilisation of TKI (all generations) post allo-HSCT for adults patients in CR1 improved OS when given as prophylactic or pre-emptive regimen. ${ }^{27}$ However, it is unclear whether this observed improvement of TKIs is significant in children. To our knowledge, our research is the first systematic review to investigate whether adding TKIs to conventional chemotherapy is indeed beneficial in paediatric Ph+ALL. Our research applied strict inclusion criteria and only those studies had comparative group of patients. Eventually, a meta-analysis was performed including one RCT and four non-RCTs with or without TKI treatment, and one RCT comparing dasatinib and imatinib in paediatric $\mathrm{Ph}+\mathrm{ALL}$.

\section{TKI versus non-TKI}

The included randomised trial EsPhALL $2004^{10}$ demonstrated that imatinib in conjunction with intensive chemotherapy might be beneficial for treating children with Ph+ALL. The 4-year DFS in good-risk patients who received intensive chemotherapy and discontinuous post-induction imatinib $300 \mathrm{mg} / \mathrm{m}^{2} /$ day increased by approximately $20 \%$ compared with patients receiving chemotherapy alone $(75.2 \%$ vs $55.9 \%)$. The long-term follow-up outcome showed 5 -year DFS in the imatinib arm of $75.5 \%$ vs $61.4 \%$ in the no imatinib arm $(p=0.20) .{ }^{28}$ However, the benefit was not significant. The pooled

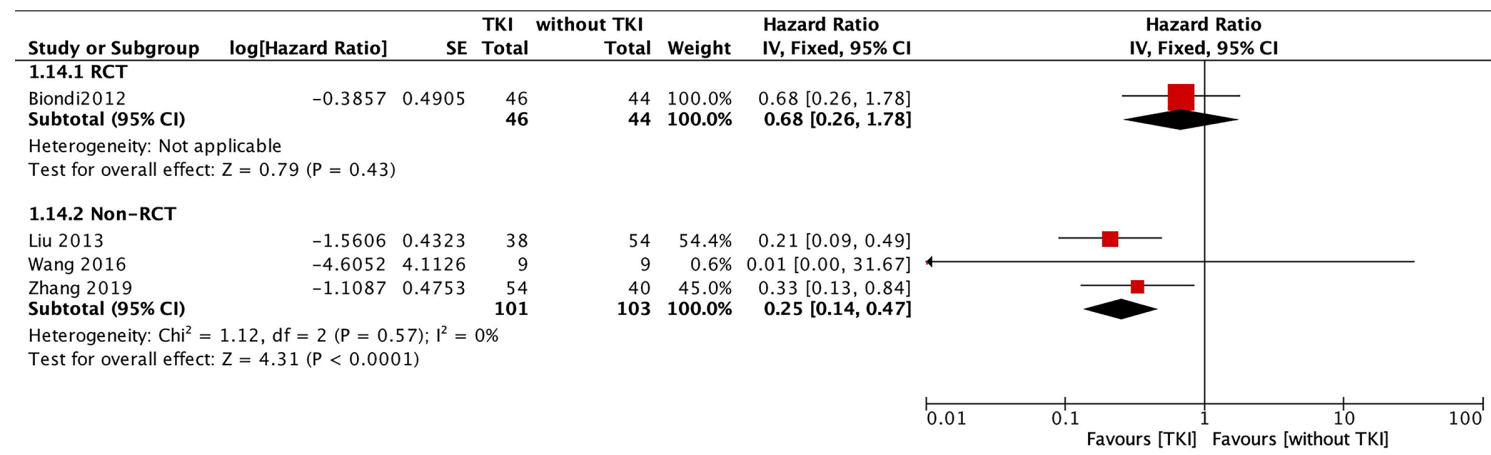

Figure 3 Meta-analysis of the effectiveness of tyrosine kinase inhibitors (TKIs) on overall survival, TKI versus non-TKI. The square data markers represent HR; horizontal lines, the $95 \%$ Cls with marker size reflecting the statistical weight of the study using fixed-effects meta-analysis. A diamond data marker represents the overall $\mathrm{HR}$ and $95 \% \mathrm{Cl}$ for the outcome of interest. $\mathrm{RCT}$, randomised controlled trial. 


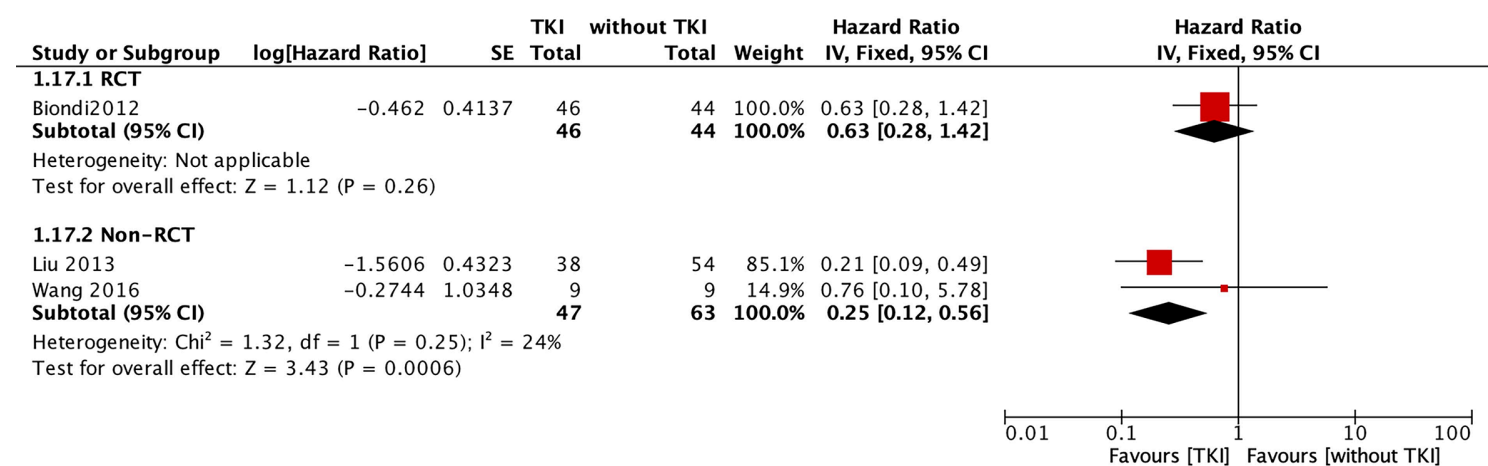

Figure 4 Meta-analysis of the effectiveness of tyrosine kinase inhibitors (TKIs) on event-free survival, TKI versus non-TKI. The square data markers represent HR; horizontal lines, the $95 \% \mathrm{Cls}$ with marker size reflecting the statistical weight of the study using fixed-effects meta-analysis. A diamond data marker represents the overall $\mathrm{HR}$ and $95 \% \mathrm{Cl}$ for the outcome of interest. $\mathrm{RCT}$, randomised controlled trial.

HR was 0.68 (95\% CI 0.26 to 1.78$)$ in OS, 0.63 (95\% CI 0.28 to 1.42 ) in EFS, respectively, comparing TKI arm with non-TKI arm in combination with intensive chemotherapy. But the results were reversed by the meta-analysis including four high-quality cohort studies (NOS score $\geq 7$ ). In these cohort studies, the pooled HR was 0.25 (95\% CI 0.14 to 0.47 ) in OS, 0.25 (95\% CI 0.12 to 0.56 ) in EFS, respectively, comparing TKI arm with non-TKI arm. The different results may be owing to the various regimen of timing and dosage of TKIs and the interference of HSCT during treatment.

In the EsPhALL 2004 study, imatinib was used at a dose of $300 \mathrm{mg} / \mathrm{m}^{2}$ for 126 days after induction. In cohort studies, imatinib $260-340 \mathrm{mg} / \mathrm{m}^{2}$ was started after induction or earlier on day 15 of remission induction. Most of the cohort studies have continued to use TKIs throughout chemotherapy unless severe infections, intolerance to TKIs or neutropenia with fever. Early and continuous exposure of imatinib may improve outcomes of OS and EFS. However, a prospective, open-label, single-arm clinical trial EsPhALL2010, the successor of EsPhALL 2004, administrated imatinib $300 \mathrm{mg} / \mathrm{m}^{2} /$ day continuously from day 15 of induction, displayed similar results to EsPhALL2004. ${ }^{29}$
The outcome of comparison between TKIs with chemotherapy versus HSCT treatment was limited in the current studies. The investigators from the COG reported the long-term outcome of paediatric Ph+ALL, showing the equivalent efficacy between the patients who received at least 280 continuous days of imatinib and those who underwent HSCT. ${ }^{9}$ Two other retrospective studies indicated that there was no significant difference in OS or EFS between HSCT and chemotherapy with TKI treatment, which was consistent with COG study. ${ }^{30}{ }^{31}$ In the included study EsPhALL 2004, ${ }^{10}$ about $80 \%$ of the patients had HSCT, 37 out of 46 in the imatinib group and 32 out of 44 in the non-imatinib group. This might lead to no statistical difference between the two groups of results in this RCT. However, the difference in the treatment outcomes of TKI combined with chemotherapy and HSCT for paediatric Ph+ALL has not been proven.

\section{Different TKIs}

In previous studies of imatinib, EFS was approximately $60 \%$ at 5 years, but resistance and relapse was common. Disappointingly, COG trial (AALL0622) for treatment of paediatric Ph+ALL did not prove the superior of dasatinib over imatinib. OS and EFS were similar in the

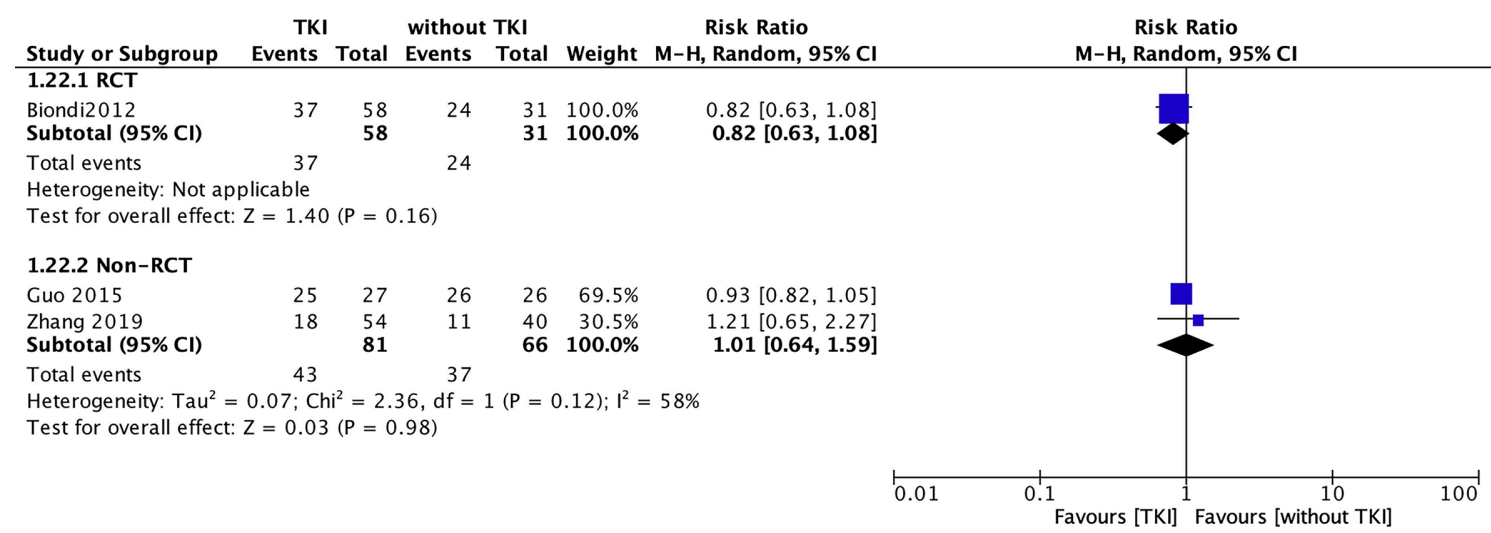

Figure 5 Meta-analysis of the safety of tyrosine kinase inhibitors (TKIs) on adverse drug reaction, TKI versus non-TKI. The square data markers represent risk ratio (RR); horizontal lines, the $95 \%$ Cls with marker size reflecting the statistical weight of the study using random-effects meta-analysis. A diamond data marker represents the overall $\mathrm{RR}$ and $95 \% \mathrm{Cl}$ for the outcome of interest. $\mathrm{RCT}$, randomised controlled trial. 
preceding trials using imatinib. ${ }^{32}$ Another COG trial (AALL1122) using dasatinib $60 \mathrm{mg} / \mathrm{m}^{2} /$ day is ongoing. ${ }^{33}$ Now, Shen $e t a l^{22}$ reported different outcomes from the first randomised trial directly comparing dasatinib with imatinib. The 4-year OS rate in the dasatinib group was significantly higher than the imatinib group $(88.4 \%$ vs $69.2 \%$; $p=0.04$ ). The 4-year EFS rate for the 92 dasatinibtreated patients was $71.0 \%$, significantly better than the $48.9 \%$ for the 97 imatinib-treated patients $(p=0.005)$. In these 189 eligible patients, there was significant difference in OS and EFS between dasatinib arm and imatinib arm (HR 2.26, 95\% CI 1.02 to 5.01; HR 2.36, 95\% CI 1.27 to 4.39 ; respectively). It is worth noting that the dose of dasatinib is higher than COG trials and longer follow-up is required to assess whether they represent significant improvement.

\section{Adverse drug reaction}

The dosing of imatinib $\left(260-570 \mathrm{mg} / \mathrm{m}^{2}\right.$.day $)$ and dasatinib (50-110 mg $/ \mathrm{m}^{2} /$ day) is well tolerated in children and adolescents with leukaemia. ${ }^{34}$ In the included studies, the daily dose of imatinib and dasatinib $260-340 \mathrm{mg} / \mathrm{m}^{2}$ and $40-80 \mathrm{mg} / \mathrm{m}^{2}$ did not increase the chemotherapyrelated toxicity and side effects such as cytopenia and pleural effusions, suggesting that imatinib or dasatinib combined with intensive chemotherapy was well tolerated. $^{36}$ There was no significance difference in severe ADR between TKI arm and without TKI arm (RR 0.82, $95 \%$ CI 0.63 to 1.08 in RCT; RR $1.01,95 \%$ CI 0.64 to 1.59 in cohort studies; respectively). Similarly, no significant difference in the frequency of severe ADR was detected between dasatinib and imatinib arm (RR $0.97,95 \%$ CI 0.77 to 1.23 ).

\section{Quality of the evidence}

The overall quality of the evidence assessed by the GRADE approach was low. Due to the rarity of Ph+ALL in this age group and the dramatic improvement in survival with TKIs reported in observational researches, data from blinded controlled studies are very scarce. In addition, the sample size was small, making the $95 \%$ CIs wide. For this reason, we limited confidence in the estimation of effect values. Further studies are likely to have an important impact on our confidence in the estimated effect and may change our estimate.

\section{Limitations}

This meta-analysis has the following limitations. First, the included studies are limited by the small study sample. In addition, HSCT is also a very important factor that needs to be taken into consideration. Even though the addition of the TKIs had improved the 5-year EFS and OS rates, but about $40 \%-80 \%$ patients received transplant. Lastly, this is a meta-analysis of prospective and retrospective studies, which has its inherent limitations. Therefore, we did not combine the results of different types of studies.

\section{CONCLUSIONS}

Overall, low-quality studies suggested that TKIs combined with intensive chemotherapy were likely to improve the OS and EFS rates in paediatric $\mathrm{Ph}+\mathrm{ALL}$, and the secondgeneration TKI dasatinib was superior to first-generation imatinib. The ADRs of TKIs could be tolerated.

This review highlights the need for future large sample size research for the use of TKIs in the treatment of paediatric $\mathrm{Ph}+\mathrm{ALL}$. Further prospective controlled studies are warranted to address remaining questions relating to the appropriate time to use TKIs during chemotherapy, as well as the role of HSCT in the management of these patients.

\section{Author affiliations}

${ }^{1}$ Department of Pharmacy/Evidence-Based Pharmacy Center, West China Second University Hospital, Sichuan University, Chengdu, Sichuan, China

${ }^{2}$ Key Laboratory of Birth Defects and Related Diseases of Women and Children, Sichuan University, Ministry of Education, Chengdu, Sichuan, China

${ }^{3}$ Department of Pediatric Hematology and Oncology, West China Second University Hospital, Sichuan University, Chengdu, Sichuan, China

${ }^{4}$ West China School of Pharmacy, Sichuan University, Chengdu, Sichuan, China

Acknowledgements We wish to thank Tingting Liang, a nuclear pharmacist from Cardinal Health in the United States, for her helping in improving the quality of English.

Contributors MC and LZ contributed to the conception of the study. The manuscript of the review was drafted by MC and revised by YZ. YL and TT independently screened the potential studies and extracted data from the included studies. YL and TT assessed the risk of bias and finished data synthesis. LZ arbitrated any disagreements and ensured that no errors occur during the review. All authors read, provided feedback and approved the final manuscript.

Funding This systematic review was funded by the Science and Technology Project of the Health Planning Committee of Sichuan (20PJ069), China.

Competing interests None declared.

Patient consent for publication Not required.

Provenance and peer review Not commissioned; externally peer reviewed.

Data availability statement All data relevant to the study are included in the article or uploaded as supplemental information. No additional data are available.

Supplemental material This content has been supplied by the author(s). It has not been vetted by BMJ Publishing Group Limited (BMJ) and may not have been peer-reviewed. Any opinions or recommendations discussed are solely those of the author(s) and are not endorsed by BMJ. BMJ disclaims all liability and responsibility arising from any reliance placed on the content. Where the content includes any translated material, BMJ does not warrant the accuracy and reliability of the translations (including but not limited to local regulations, clinical guidelines, terminology, drug names and drug dosages), and is not responsible for any error and/or omissions arising from translation and adaptation or otherwise.

Open access This is an open access article distributed in accordance with the Creative Commons Attribution Non Commercial (CC BY-NC 4.0) license, which permits others to distribute, remix, adapt, build upon this work non-commercially, and license their derivative works on different terms, provided the original work is properly cited, appropriate credit is given, any changes made indicated, and the use is non-commercial. See: http://creativecommons.org/licenses/by-nc/4.0/.

ORCID iD

Lingli Zhang http://orcid.org/0000-0002-9507-4496

\section{REFERENCES}

1 Schlieben S, Borkhardt A, Reinisch I, et al. Incidence and clinical outcome of children with Bcr/Abl-positive acute lymphoblastic leukemia (all). A prospective RT-PCR study based on 673 patients 
enrolled in the German pediatric multicenter therapy trials ALLBFM-90 and CoALL-05-92. Leukemia 1996;10:957-63.

2 Reiter A, Schrappe M, Ludwig WD, et al. Chemotherapy in 998 unselected childhood acute lymphoblastic leukemia patients. results and conclusions of the multicenter trial ALL-BFM 86. Blood 1994;84:3122-33.

3 Ribeiro RC, Broniscer A, Rivera GK, et al. Philadelphia chromosomepositive acute lymphoblastic leukemia in children: durable responses to chemotherapy associated with low initial white blood cell counts. Leukemia 1997;11:1493-6.

4 Aricò M, Schrappe M, Hunger SP, et al. Clinical outcome of children with newly diagnosed Philadelphia chromosome-positive acute lymphoblastic leukemia treated between 1995 and 2005. J Clin Oncol 2010;28:4755-61.

5 Aricò M, Valsecchi MG, Camitta B, et al. Outcome of treatment in children with Philadelphia chromosome-positive acute lymphoblastic leukemia. N Engl J Med 2000;342:998-1006.

6 Ravandi F. How I treat Philadelphia chromosome-positive acute lymphoblastic leukemia. Blood 2019;133:130-6.

7 NCCN, Clinical Practice Guidelines in Oncology. Acute lymphoblastic leukemia. secondary clinical practice guidelines in oncology. Acute Lymphoblastic Leukemia, 2018. https://www.nccn.org/professionals/ physician_gls/default.aspx

8 Schultz KR, Bowman WP, Aledo A, et al. Improved early event-free survival with imatinib in Philadelphia chromosome-positive acute lymphoblastic leukemia: a Children's Oncology Group study. J Clin Oncol 2009;27:5175-81.

9 Schultz KR, Carroll A, Heerema NA, et al. Long-term followup of imatinib in pediatric Philadelphia chromosome-positive acute lymphoblastic leukemia: Children's Oncology Group study AALL0031. Leukemia 2014:28:1467-71.

10 Biondi A, Schrappe M, De Lorenzo P, et al. Imatinib after induction for treatment of children and adolescents with Philadelphiachromosome-positive acute lymphoblastic leukaemia (EsPhALL): a randomised, open-label, intergroup study. Lancet Oncol 2012;13:936-45.

11 Brave M, Goodman V, Kaminskas E, et al. Sprycel for chronic myeloid leukemia and Philadelphia chromosome-positive acute lymphoblastic leukemia resistant to or intolerant of imatinib mesylate. Clin Cancer Res 2008;14:352-9.

12 Kantarjian H, Shah NP, Hochhaus A, et al. Dasatinib versus imatinib in newly diagnosed chronic-phase chronic myeloid leukemia. $N$ Engl $J$ Med 2010;362:2260-70.

13 O'Hare T, Walters DK, Stoffregen EP, et al. In vitro activity of Bcr-Abl inhibitors AMN107 and BMS-354825 against clinically relevant imatinib-resistant Abl kinase domain mutants. Cancer Res 2005;65:4500-5.

14 Porkka K, Koskenvesa P, Lundán T, et al. Dasatinib crosses the blood-brain barrier and is an efficient therapy for central nervous system Philadelphia chromosome-positive leukemia. Blood 2008;112:1005-12.

15 Cortes JE, Kantarjian H, Shah NP, et al. Ponatinib in refractory Philadelphia chromosome-positive leukemias. N Engl J Med 2012;367:2075-88

16 Nicolini FE, Basak GW, Kim D-W, et al. Overall survival with ponatinib versus allogeneic stem cell transplantation in Philadelphia chromosome-positive leukemias with the T315I mutation. Cancer 2017;123:2875-80.

17 Higgins JGS. Cochrane Handbook for systematic reviews of interventions. Chichester, UK: The Cochrane Library, John Wiley \&Sons, 2020. https://methodscochraneorg/risk-bias-20-tool

18 Wells GA SB, O'Connell D. The Newcastle-Ottawa scale (NOS) for assessing the quality if nonrandomized studies in meta-analyses. Available: http://www.ohri.ca/programs/clinical_epidemiology/oxford. asp [Accessed 10 Sep 2020].

19 Parmar MK, Torri V, Stewart L. Extracting summary statistics to perform meta-analyses of the published literature for survival endpoints. Stat Med 1998;17:2815-34.
20 Review Manager (RevMan) [Computer program]. Version 5.3.Copenhagen: the Nordic Cochrane centre. The Cochrane Collaboration, 2014.

21 Guyatt G, Oxman AD, AkI EA, et al. Grade guidelines: 1. IntroductionGRADE evidence profiles and summary of findings tables. $J$ Clin Epidemiol 2011;64:383-94.

22 Shen S, Chen X, Cai J, et al. Effect of dasatinib vs imatinib in the treatment of pediatric Philadelphia chromosome-positive acute lymphoblastic leukemia: a randomized clinical trial. JAMA Oncol 2020;6:358-66.

23 Zhang J, ZF L, Guo J. Clinical observation of inductive treatment of childhood acute lymphoblastic leukemia with imatinib mesylate combined with VDLD Regimen. Clinical Misdiagnosis \& Mistherapy 2019;32:26-30.

24 Wang CC, Jin RM. Clinical analysis of 19 BCR-ABL positive childhood acute lymphoblastic leukemia. Master dissertation. Huazhong University of Science and Technology 2016.

25 Guo Y, Liu T-F, Ruan M, et al. [Efficacy and safety of imatinib for the treatment of Philadelphia chromosome-positive acute lymphoblastic leukemia in children]. Zhongguo Dang Dai Er Ke Za Zhi 2015;17:819-24.

26 Liu TF, Zhu XF. Clinical and biological characteristics, therapeutic effects and prognostic factors of 92 children with $\mathrm{PhpH}$ chromosome positive acute lymphoblastic leukemia [Doctor dissertation]. Peking Union Medical College, 2013.

27 Warraich Z, Tenneti P, Thai T, et al. Relapse prevention with tyrosine kinase inhibitors after allogeneic transplantation for Philadelphia chromosome-positive acute lymphoblast leukemia: a systematic review. Biol Blood Marrow Transplant 2020;26:e55-64.

28 Biondi A, Cario G, De Lorenzo P, et al. Long-Term follow up of pediatric Philadelphia positive acute lymphoblastic leukemia treated with the EsPhALL2004 study: high white blood cell count at diagnosis is the strongest prognostic factor. Haematologica 2019;104:e13-16.

29 Biondi A, Gandemer V, De Lorenzo P, et al. Imatinib treatment of paediatric Philadelphia chromosome-positive acute lymphoblastic leukaemia (EsPhALL2010): a prospective, intergroup, open-label, single-arm clinical trial. Lancet Haematol 2018;5:e641-52.

30 Salami K, Alkayed K, Halalsheh H, et al. Hematopoietic stem cell transplant versus chemotherapy plus tyrosine kinase inhibitor in the treatment of pediatric Philadelphia chromosome-positive acute lymphoblastic leukemia (all). Hematol Oncol Stem Cell Ther 2013;6:34-41.

31 Gao Y-J, Guo Y, Hu S-Y, et al. Philadelphia chromosome-positive acute lymphoblastic leukemia in China: a retrospective study from the Chinese childhood cancer group. Leuk Lymphoma 2016;57:2696-8.

32 Slayton WB, Schultz KR, Kairalla JA, et al. Dasatinib plus intensive chemotherapy in children, adolescents, and young adults with Philadelphia chromosome-positive acute lymphoblastic leukemia: results of children's Oncology Group trial AALL0622. J Clin Oncol 2018;36:2306-14.

33 ClinicalTrials.gov. Pediatric Philadelphia positive acute lymphoblastic leukemia. Available: https://www.clinicaltrials.gov/ct2/show/ NCT01460160?term=AALL1122\&draw $=2 \& r a n k=1$ [Accessed $10 \mathrm{Jul}$ 2020].

34 Champagne MA, Capdeville R, Krailo M, et al. Imatinib mesylate (STI571) for treatment of children with Philadelphia chromosomepositive leukemia: results from a Children's Oncology Group phase 1 study. Blood 2004;104:2655-60.

35 Aplenc R, Blaney SM, Strauss LC, et al. Pediatric phase I trial and pharmacokinetic study of dasatinib: a report from the Children's Oncology Group phase I Consortium. J Clin Oncol 2011;29:839-44.

36 Lindauer M, Hochhaus A. Dasatinib. Recent Results Cancer Res 2018;212:29-68. 